\title{
Comparative Transcriptomic Analysis Provides Insight Into Carpel Petaloid in Lotus (Nelumbo Nucifera)
}

Zhongyuan Lin ( $\sim$ lzy2019@mju.edu.cn )

Minjiang University

Dingding Cao

Minjiang University

Rebecca Njeri Damaris

Hubei University

Pingfang Yang

Hubei University

Research Article

Keywords: Nelumbo nucifera, Flower morphology, Petaloid, Floral organ, MADS box

Posted Date: February 19th, 2021

DOI: https://doi.org/10.21203/rs.3.rs-199154/v1

License: (9) This work is licensed under a Creative Commons Attribution 4.0 International License.

Read Full License 


\section{Abstract}

Background: Lotus (Nelumbo nucifera) is a famous flower with high ornamental value. Flower color and flower morphology are two main factors for flower lotus breeding. Petaloid is a universal phenomenon in lotus flowers. However, the genetic regulation of floral organ petaloid in lotus still remains unclear.

Results: In this study, the transcriptomic analysis was performed among five organs, including petal, stamen petaloid, stamen, carpel petaloid, and carpel in lotus. 812 annotated DEGs related to carpel petaloid were identified. Our study showed that DNA methylation may be involved in regulating carpel petaloid. To identify the patterns of gene expression in the petaloid process using WGCNA analysis, 37 candidate genes were found to be related to carpel petaloid. Additionally, one floral homeotic gene encoded by MADS box transcription factor, $\operatorname{AGAMOUS}(A G)$, was identified as the candidate gene for petaloid in lotus. Meanwhile, a predicted labile boundary in floral organs of $N$. nucifera was hypothesized.

Conclusions: In summary, the above results explored the candidate genes related to carpel petaloid, setting a theoretical basis for the molecular regulation of petaloid phenotype.

\section{Background}

Lotus (Nelumbo nucifera) is an aquatic plant, which is widely cultivated as food crop in East Asia. Additionally, it is also one of the famous traditional flowers, especially in China. Based on different breeding purposes, lotus is classified into three groups, namely seed lotus, rhizome lotus, and flower lotus. With its high ornamental value, the aim of breeding in flower lotus is performed under distinct flower colors and shapes. Generally, a flower is constituted by four floral organs, including sepal, petal, stamen, and carpel in angiosperm plant. Thus, the different number of floral organs and diverse organs features form various flower morphologies. Meanwhile, few-petalled, double-petalled, duplicate-petalled, and all-double-petalled flowers were classified in lotus flower morphology [1, 2]. Seed lotus and rhizome lotus generally have normal floral patterns with few-petalled. The seed lotus flower has a normal pistil advantaged for seed harvesting while the rhizome lotus favors underground development with a few flowers. In particular, flowers with aberrant floral organs are preferred for ornamental purposes as they consist of all the classifications of flower morphology. In lotus, the peculiar flower patterns are mainly constructed by aberrant floral organs, such as stamen petaloid and carpel petaloid.

The petaloid phenomenon attracted research attention as early as $286 \mathrm{BC}$ [3]. Petaloid organs in other locations other than the flower have been discovered and having petal-like morphology [4]. Based on three floral homeotic mutations and genetic relationships between Arabidopsis and Antirrhinum, the typical ABC model has been widely accepted since the 1990s $[5,6]$. Based on this model, petals are determined by $A$ and $B$ class genes; stamens are determined by $B$ and $C$ class genes; while carpels are determined by $\mathrm{C}$ class genes. Most of the $\mathrm{ABC}$ model genes are MADS box transcription factors, except one A class gene encoded by APETALA2 (AP2). Meanwhile, this model is also applicable to many monocot flowers after modifications, despite their differences in flower morphology $[7,8]$. B-function 
genes, including APETALA3/PISTILLATA (AP3/PI) are essential in influencing petaloid [7]. The role of $A P 3 / P / g e n e s$ is specification in 'petaloid' traits under the sliding borders or fading borders models, which is different from the earlier models [4]. $C$ and $A$ function genes have antagonism regulation [9]. Loss of $C$ class genes functions results in the substitution of petals for stamens and sepals for carpels [10]. The reduction in the expressions of $\operatorname{AGAMOUS}(A G)$, a C-function MADS box gene, homolog gene caused the double flower morphology in rose, Thalictrum thalictroides, and Cyclamen persicum [11-13]. When the expression of RABBIT EARS (RBE) is down-regulated, the transcripts of $A G$ are derepressed in floral and inflorescence meristems [14]. The interactions between WUSCHEL (WUS) and $A G$ are involved in floral determination while $A G$ is a central gene in the genetic network of floral organ development [15]. During flower patterning, $A P 3$ and $A G$ associate with $\angle E A F Y(L F Y)$ after induction [16]. Other transcription factors might regulate the floral organ formation through affecting the $A B C$ model genes. However, the molecular mechanism of petaloid is still not fully understood.

Previously, it was shown that the obscure expression of several candidate genes in boundaries of petal and stamen could result in the stamen petaloid formation [17], which might also be influenced by DNA methylation [18]. The transgenic lines of $A P 3$ promoter with hypermethylation possessed abnormal stamens and petals [19]. DNA hypermethylation inhibits the expression of $R h A G$ that controls petal number [20]. DNA methylation affects plantdevelopment via gene regulation and silencing of the trasnsposable element [21]. Altered DNA methylation shows diverse phenotypes, like altered floral morphologies [22]. Additionally, the latest studies on lotus were comprehensively reviewed which revealed the absence of a detailed study on petaloid formation in N. nucifera [23].

To obtain a comprehensive understanding of the petaloid formation in lotus, we used transcriptomic analyses among petal (P), stamen petaloid (Sp), stamen (St), carpel petaloid (Cp), and carpel (C) from a bowl lotus 'Sleeping Beauty'. The obtained results might provide some new insights into improving our understanding of petaloid formation.

\section{Results}

\section{Petaloid phenotype of $\boldsymbol{N}$. nucifera}

Lotus is a famous aquatic flowering plant, which has high ornamental value. The flowers of lotus are beautiful with gorgeous colors and various flower morphologies. With artificial selection, double flower is popular for the landscape architecture. Among them, 'Sleeping beauty' is a bowl lotus with long flowering time and abundant number of flowers, which also possess floral aberration. During different flowering stages, a special phenomenon in the flower shape of 'Sleeping beauty' occurs (Fig. 1). The abnormal floral organs are stamen petaloid and carpel petaloid, which are defective for reproduction (Fig. 1). Scanning electron microscope was used to visualize the epidermal cell morphology of petal, stamen petaloid and carpel petaloid. The upper and lower epidermal cells were shown to have similar shapes, including mastoid cells and wax crystal, among P, Sp, and Cp (Fig. 2).

\section{Overview of the transcriptomic analysis}


RNA-seq was performed for five samples, including $\mathrm{P}, \mathrm{Sp}, \mathrm{St}, \mathrm{Cp}$, and $\mathrm{C}$, with each one having three biological replicates (Fig. 1). Under sequencing quality control, a total of $50.4 \mathrm{~Gb}$ clean data was generated. The percentage of Q30 in each sample was no less than $91.61 \%$ (Table S1). 85.52-94.24\% clean reads of each sample were mapped to the lotus genome [24]. The total number of genes or transcripts from the samples was 30469, out of which 3784 were noted as new genes (Table S2). After filtering out the genes with a low expression (FPKM < 5), DEGs were screened based on an absolute fold change of no less than two and an FDR $\leq 0.01$. A total of 8238 (C vs P), 3944 (C vs Cp), 4481 (P vs Cp), 4231 (Cp vs Sp), 216 (P vs Sp), 1223 (St vs Sp), and 2450 (St vs P) DEGs were detected, including 3637, 2133, 2932, 1779, 199, 821, and 1095 up-regulated DEGs and 4601, 1811, 1549, 2452, 17, 402, and 1355 down-regulated DEGs, respectively (Fig. 3). Moreover, the Pearson relationships were performed with pairwise comparison among these five tissues with their DEGs (Fig. S1). We found that P vs Sp had the highest relationship up to 0.9237 , while $\mathrm{C}$ vs $\mathrm{P}$ had the lowest relationship down to 0.6238 (Fig. S1). The result suggested that petal and stamen petaloid are highly similarity.

To verify the reliability of RNA-seq data, fifteen DEGs were selected and subjected to qRT-PCR analysis in five floral organs (including P, Sp, St, Cp, and C). Most of the selected genes exhibited similar trend with RNA-seq data, except for the NNU_17837 and NNU_21294 (but their R was still more than 0.75, Fig. 4). Meanwhile, the comparison of the DEGs in Petal, Stamen petaloid, and Stamen of 'Sleeping beauty' from previous study showed 1140 common DEGs. Their correlation relationship was 0.8160 (Fig. S2). These results further proved the reliability of the transcriptome data.

\section{Identification of DEGs involved in carpel petaloid}

$\mathrm{P}, \mathrm{Sp}, \mathrm{St}, \mathrm{Cp}$, and $\mathrm{C}$ were divided into two comparison pairs groups, including the carpel petaloid group (C vs $P, C$ vs $C p$, and $P$ vs $C p$ ) and stamen petaloid group (St vs P, P vs Sp, and St vs Sp). Between the carpel petaloid group and stamen petaloid group, there were seven common DEGs including NNU_04669, NNU_09105, NNU_10192, NNU_21294, NNU_22371, NNU_23867, and NNU_26585. It is suggested that they are involved in petaloid formation. Since the stamen petaloid group had been reported previously [17], the carpel petaloid group was focused to investigate the regulation of gene expression in the carpel petaloid in this study. A total of 10433 DEGs were found in carpel petaloid group. But only $9.82 \%$ of the DEGs (containing 1025 common genes) were common in these floral organs being selected (Fig. 3). Furthermore, the number of DEGs that could be annotated was only 812 from the common DEGs in the different flower organs. The hierarchical clustering analysis of their gene expression presented differentiated profiles in the carpel petaloid group, with three large gene clusters (Fig. 5). The largest cluster (cluster 1 highlighted in green) consisted of 411 genes; while the cluster 2 (highlighted in blue) contained 281 genes. Their expressions in $\mathrm{Cp}$ were median level compared with $\mathrm{C}$ and $\mathrm{P} ; 120$ genes (highlighted in red) were grouped in cluster 3 with most of them expressed more strongly in Cp (Fig. 5A). Annotation, GO-based and KEGG-based functional ananlyses were performed (Fig. S3 and Fig. S4). For all the chosen DEGs, DNA methylation and histone H3-K9 methylation were the top two most enriched GO terms (Fig. 5B). Otherwise, carbon metabolism, phenylpropanoid biosynthesis, starch and sucrose metabolism, photosynthesis, and plant hormone signal transduction were the five top classifications (Fig. 
5C). Regulation of DNA replication, cellular response to light stimulus, and chloroplast thylakoid membrane were the most enriched GO term in cluster 1, cluster 2, and cluster 3, respectively (Fig. S3). Meanwhile, annotated genes by KEGG classification included ubiquitin mediated proteolysis, biosynthesis of amino acids, carbon metabolism, starch and sucrose metabolism, and flavonoid biosynthesis in cluster 1 (Fig. S4). For cluster 2, there were plant hormone signal transduction, phenylpropanoid biosynthesis, glycerolipid metabolism, fatty acid degradation, and glyoxylate and dicarboxylate metabolism (Fig. S4). Cluster 3 was contained photosynthesis, photosynthesis-antenna proteins, carbon metabolism, carbon fixation in photosynthetic organisms, and starch and sucrose metabolism (Fig. S4).

From the 812 DEGs, the majority of them were assigned to plant hormone signal transduction and transcription factors (TFs) (Fig. S5 and Table S3). 33 DEGs were involved in phytohormone regulation (Fig. S5A). They were assigned as the auxin (AUX), abscisic acid (ABA), jasmonic acid (JA), cytokinine (CTK), gibberellin (GA), brassinosteroid (BR), ethylene (ETH), and salicylic acid (SA) pathways. Those related to AUX regulation pathway were the most enrichment, suggesting that AUX pathway may play a significant role in carpel petaloid. Notably, only twelve assigned as plant hormone signal transduction by KEGG pathway annotation. Seven of them were shown to be involved in AUX pathway, namely, five AUX/IAA (NNU_05583, NNU_10534, NNU_14285, NNU_19926, and NNU_26265) and two GH3 genes (NNU_08907 and NNU_22327). They were considerably varied in expression in the carpel petaloid group. The expression patterns of five out of seven genes in carpel petaloid group were similar and peaked in petal, while NNU_10534 had an opposite pattern. Interestingly, a gene encoding Indole-3-acetic acidamido synthetase GH3 (NNU_22327) was highly expressed in carpel petaloid.

In our data, there were 47 TFs out of the 812 DEGs in the pair comparison of the carpel petaloid group (Fig. S5B). Interestingly, five DEGs encoding MADS-box domain were found. Four of them were belonged to classical ABC model. They were three class B genes included NNU_02674 (PI), NNU_08090 (AP3), and NNU_23351 (AP3). Additionally, NNU_10192 being an AG homologue gene, a member of Class C members had interesting expression patterns revealed by the FPKM value via transcriptome profiling in different floral organs (Fig. 6). NNU_10192 and NNU_26656 encoding for floral homeotic AG gene were found to have the lowest expression in petal compared with other flower tissues. In contrast, A class genes (NNU_04430, NNU_17043, and NNU_13608) and B class genes (NNU_08090, NNU_23351, and NNU_02674) expressed higher in petal. Additionally, based on the expression profiles (Fig. 6), A class genes, AP1-like (NNU_04430) and AP2-like(NNU_17043) had a similar expression, being down-regulated in C vs Cp and St vs Sp. C class genes (NNU_10192 and NNU_26656) showed the opposite expression.

\section{Weighted genes co-expression network analysis (WGCNA)}

To identify the patterns of gene expression in petaloid process, WGCNA was performed for analyzing weighted gene co-expression network aimed at further understanding the floral organ formation in lotus. Six modules were obtained, including grey, green, turquoise, yellow, blue, and brown (Fig. 7A). Thereinto, the grey module was a collection of genes that could not be assembled into other modules and had 836 
genes. There were 1185, 7527, 1857, 3615 and 2595 genes in green, turquoise, yellow, blue, and brown module, respectively. The green module was found to be related to carpel petaloid, of which module-trait relationships value was 0.96 (Fig. 7A and B). 360 hub genes (module-trait relationships value >0.9) were selected of which 81 genes were both hub genes and DEGs (Fig. 7C). Among them, 37 genes $\left(\log _{2} \mathrm{FC}>5\right.$ or $<-5$ ) were chosen as candidate genes including five transcription factors COL16 (NNU_00499), bHLH51 (NNU_13078), MYB38 (NNU_19814), GATA9 (NNU_23627), and bHLH35 (NNU_26108) (Fig. 7D). One of them is related to plant auxin gene GH3 (NNU_22327). Additionally, other three genes including NNU_01046, NNU_12218, and NNU_21373 are the members of P450 family.

\section{Discussion}

The various phenotypes of lotus flowers have fundamental own critical ornamental value. The morphology of a flower is influenced by the aberrant floral organs, especially petaloid. Cultivar lotus 'Sleeping Beauty' has various abnormal floral organs similar to petals from the homeotic transformation of stamen and carpel. To explore the mechanism of petaloid formation, comparative transcriptomic analysis was performed in $\mathrm{P}, \mathrm{Sp}, \mathrm{St}, \mathrm{Cp}$, and $\mathrm{C}$. This will enable the expansion of our understanding of flower development and petaloid formation in lotus.

Currently, two lotus genome including 'China Antique' and 'Chinese Tai-zi' had been sequenced and released providing a research basis for omic study and breeding $[24,25]$. Through transcriptomic analysis in $\mathrm{P}, \mathrm{Sp}, \mathrm{St}, \mathrm{Cp}$, and C, DEGs involved in petaloid were identified. In the pairwise comparison of petal, stamen petaloid, and stamen, DEGs in P vs Sp were fewer than those in St vs Sp (Fig. 3). It has been declared that stamen petaloid is more similar to petal than to stamen, which is consistent with stamen petaloid having the petal morphology as previously reported [17]. The number of DEGs between C vs Cp and $\mathrm{P}$ vs $\mathrm{Cp}$ did not show any significant difference, suggesting that the abnormal flower organ (carpel petaloid) remained much of the carpel trait with petal-like features.

Using WGCNA, genes implicated in carpel petaloid were screened, including transcription factors and hormone-related genes (Fig. 7). Previously, it was reported that not only MYB21 and MYB24 improve petal and stamen development, but also induce carpel growth [26]. Here, NNU_19814, which is a MYB38 ortholog gene, showed the highest level in the carpel petaloid, suggesting it's being involved in petal development. Different stages of flower development are controlled by $b H L H$ gene, including regulating the growth of carpel margin tissues [27]. In lotus, $117 \mathrm{bHLH}$ genes were identified with most of their functions have not yet been confirmed [28]. In our study, two bHLH homologue genes, NNU_13078 and NNU_26108, were specifically expressed in carpel petaloid. HAN encodes a GATA transcription factor that regulates floral organ specification that directly controlling hormone response genes and correlates with the number of petals $[29,30]$. GH3.5 is a direct downstream gene of $H A N$, whose mutant exhibits low transcript of $G H 3.5$ [29]. This idea is consistent with our result that the expression profile of $G H 3$ (NNU_22327) is similar to GATA4 (NNU_22327) and is significantly expressed in the carpel petaloid of $N$. nucifera. These candidate genes were reported to be involved in stamen petaloid [17]. This indicates that $\mathrm{GH} 3$ and GATA are essential in petal formation and promote to the alteration of other floral organs to 
petal-like features. Additionally, the $G H 3$ gene is regulated by phytochrome $\mathrm{B}$, and modulates the light signaling pathway [31]. CONSTANTS-LIKE (COL) gene homolog NNU_00499, which is annotated as COL 16 belongs to a COL family of zinc finger protein transcription factor. A previously reported COL 16 in petunia is involved in the chlorophyll biosynthesis [32]. CYP715 is a homolog of cytochrome P450 family members, which is a key regulator of floral maturation, affecting petal development and maintains flower hormone homeostasis [33]. Overexpression of $K L U H / C Y P 78 A 5$ leads to an increase in the number of petals and epidermis cells, while they decrease in klu/cyp78a5 mutant. This suggests that KLUH/CYP78A5 regulates organ development via cell proliferation [34, 35]. Three genes encoding a cytochrome P450, including NNU_01046, NNU_12218 and NNU_21373, had a similar expression pattern, suggesting that they are possibly involved in the regulation of petaloid formation. ANS (NNU_08856) and ANR (NNU_08935) referred to as the flavonoids synthesis pathway was found by transcriptomic analysis. The results potentially associate with the surface of carpel petaloid with light green color (Fig. 1D). Glyceraldehyde-3-phosphate dehydrogenase GAPCP2 (NNU_01252), which is one of the glycolysis/gluconeogenesis pathway genes being related to carbohydrate transport and metabolism plays a key role in maintaining reproductive organs development [36]. Further study on glyceraldehyde-3phosphate dehydrogenase effects on alteration of carpel to petal needs to be carried out. For the above candidate genes, they are almost certainly involved in the regulatory network of flower development but their connections with floral organ formation have not been verified.

In model plants, a large number of transcription factors were reported to be involved in floral development, such as MADS-box, MYB, and bHLH [37-39]. MADS-box transcription factors play key roles in controlling morphogenesis of floral organs. AGAMOUS $(A G)$ is involved in the regulation of stamen and carpel formation and development in Arabidopsis [10,40]. Owing to A class genes being expanded to inner whorl, the transformation of stamens to petals was as a result of mutation of $A G$. In contrast, ectopic expression of $A G$ in outer whorl causes sepal carpeliod and petal stamenoid [41]. $A G$ homeotic gene has been universally identified in many plants, such as rose, petunia, Thalictrum thalictroides, Prunus lannesiana and Medicago truncatula [11, 13, 42-44]. These show that the homologous $A G$ pattern of expressions and regulation of stamen and carpel identity are conservative. In our study, notably, the candidate gene (NNU_10192) is an $A G$ homolog, belonging to MADS-box family member. $A G$ was among the DEGs with lower expression in stamen petaloid than in stamen. Meanwhile, its expression in carpel petaloid was less than that in carpel and lowest in petal (Fig. 6). These results show that a declined expression of $A G$ in the inner whorl results in carpel petaloid formation and breaks down the gene expression boundary. In the previous study, we performed transcriptome analysis for stamen petaloid showing that several MADS-box genes, including $A G$ were found to be possibly involved in floral organ specification [17]. Epigenetic regulation may influence carpel petaloid formation (Fig. 5B). DNA methylation and histone methylation may transmit genetic information through synergic action. The DNA methylation level of $R h A G$ regulated its expression to determine the petal number [20]. DNA methylation status was different in three floral organs of lotus [18]. The dynamics of DNA methylation controlled floral organ petaloid and their activated pathways will be carried out in future studies. 
A few-petalled, double-petalled, duplicate-petalled, and all-double-petalled groups were systematized for the flower morphologies in lotus [1]. The different number of petal in cultivar lotus was generated by breeder domestication. For traditional breeding and production, they only focus on their aims of improving plants potential values. Factors in the molecular mechanisms on how modulated is still unclear. In normal floral morphology, dicotyledonous plants' floral organs are located in four whorls in an orderly manner. Boundaries exhibit between floral organs within whorls [45]. The labile boundary was previously found in rose flower and that the expression pattern of $A G$ is responsible for morphological diversity [11].

A number of candidate targets of MADS box genes have known function in petal growth [46]. In the floral classic $A B C$ model, petal identity is specified by $A$ and $B$ gene classes, stamen by $B$ and $C$ gene classes and carpel by $C$ gene classes. From Fig. 6 , our results suggest that $A, B$ and $C$ class genes are involved in petaloid formation, which is in agreement with previous studies. Deficiencies in inter-whorl boundaries can result in hybrid structures such as petal-stamens [47]. The stamen petaloid and carpel petaloid in lotus may also be caused by defects in inter-whorl boundaries. In accordance with this supposition, the gene model of lotus flower was summarized in Fig. 8. Our hypothesis proposed that not only boundary between $A$ and $C$ class genes was shift, but also the boundary of $B$ and $C$ class genes was changed in double flower of louts. The boundary genes should be conducted to understand how they build the restricted expression pattern and perform functions in their complicated regulation network.

\section{Conclusions}

This study was carried out to investigate the different transcriptomic dynamics resulting in abnormal flower morphology of lotus. Through comprehensive analysis, 812 annotated DEGs related to carpel petaloid were identified. Fifteen DEGs were validated by qRT-PCR. Several transcription factors and multiple genes involved in phytohormone pathways were found associated with carpel petaloid formation in this study. Notably, the member of the MADS-box family, $A G$ being a floral homeotic gene played a key role in floral organ petaloid.

\section{Materials And Methods}

\section{Plant growth and sample collection}

Lotus cultivar 'Sleeping beauty' was acquired from field genebank for lotus in Wuhan Botanical Garden, Chinese Academy of Sciences (WBGCAS), Hubei Province, China. The rhizomes of 'Sleeping beauty' were then separated into three plastic buckets $(90 \mathrm{~cm} \times 90 \mathrm{~cm})$. Petal, stamen petaloid, stamen, carpel, and carpel petaloid were collected when the flowers bloomed (Fig. 1). After sampling, the floral organs were snap frozen in liquid nitrogen and stored at $-80{ }^{\circ} \mathrm{C}$ until RNA and protein extraction.

\section{Scanning electron microscope (SEM) observation}


Petal, stamen petaloid, and carpel petaloid were collected from cultivar lotus 'Sleeping Beauty'. SEM was used to view epicuticular cells. Samples were prepared and imaged by SEM as described by Lü et al. [48].

\section{RNA isolation and quantitative real-time PCR (qRT-PCR) gene expression analysis}

Total RNAs were isolated using an RNA reagent (OminiPlant RNA Kit, CWBIO, China), and genomic DNA contamination was removed by treating with RNase-free DNasel (Thermo, Shanghai, China). Primers used for qRT-PCR were listed in Table S4. The qRT-PCR reactions were performed as described by Lin et al. [17].

\section{Sequencing, data processing, and bioinformatic analysis}

For each sample, more than $1.5 \mu \mathrm{g}$ of total RNA was used. The RNA integrity number (RIN) of each sample was set above 6.5. Fifteen cDNA libraries were constructed, and Illumina sequenced by Beijing Novogene Bioinformatics Technology Co., Ltd. using the Illumina HiSeq 2500 high throughput sequencing platform. The transcriptome sequencing data was deposited in PRJNA524054.

After quality control, clean data was deposited in BMKCloud (https://www.biocloud.net/) for analysis. We mapped the data to the reference genome of Nelumbo nucifera (China lotus 1.1) [24] using TopHat2 Software v2.0.9 [49]. Transcript assembly, differential expression, and divergent regulation were performed using Cufflinks v2.1.1 [50]. The differentially expressed genes (DEGs) were carried out by using DESeq package (http://bioconductor.org/packages/release/bioc/html/DESeq.html). The expression (read counts) was calculated by RSEM v1.2.15 [51]. The DEGs were identified by false discovery rate (FDR) < 0.01 and a fold change $\geq 2$. Any genes with an adjusted $P$-value $<0.05$ were assigned as differentially expressed. The Gene Ontology (GO) enrichment analysis of DEGs was carried out by the GOseq R packages 1.10.0. The statistical enrichment of DEGs in Kyoto Encyclopedia of Genes and Genomes (KEGG) pathways (FDR $\leq 0.05$ ) was tested by KOBAS (KEGG Orthology-Based Annotation System) software. The heatmap was constructed by using Multiple Experiment Viewer software (MeV 4.9.0, https://sourceforge.net/projects/mev-tm4/files/mev-tm4/MeV\%204.9.0/). Weighted gene co-expression network analysis (WGCNA) was applied for identification of co-expressed genes by WGCNA package in the R software. The hub genes were further grouped into six modules using WGCNA.

\section{Declarations}

\section{Funding}

This work was supported by talents project to associate Prof. Zhongyuan Lin from Minjiang University and distinguished talents project to Prof. Pingfang Yang from Hubei University.

\section{Author contributions}

ZL and FY designed the experiments. ZL contributed to data analysis and wrote the manuscript. ZL, DC, and ND performed the experiments. DC, ND and FY revised the manuscript. All authors commented on the 
manuscript.

\section{Availability of data and materials}

The RNA-seq data generated in this study are available in the NCBI using accession numbers PRJNA524054.

\section{Competing interests}

The authors declare that they have no competing interests.

\section{Acknowledgements}

Not Applicable

\section{Consent for publication}

Not applicable

\section{Ethics approval and consent to participate}

Not applicable.

\section{Acknowledgements}

This work was supported by talents project to associate Prof. Zhongyuan Lin from Minjiang University and distinguished talents project to Prof. Pingfang Yang from Hubei University.

\section{Abbreviations}

ABA: Abscisic acid; AG: AGAMOUS; AP: APETALA; AUX: auxin ; BR: Brassinosteroid; C: Carpel; Cp: Carpel petaloid; CTK: Cytokinine; DEGs: Differentially expressed genes; ETH: Ethylene; FDR: False discovery rate; FPKM: Fragments per kilo base of transcript per million base pairs sequenced; GA: Gibberellin; GO: Gene ontology; JA: Jasmonic acid; KEGG: Kyoto encyclopedia of genes and genomes; KOBAS: KEGG orthologybased annotation system; LFY: LEAFY; MADS: MCM1, AG, DEFA and SRF; P: Petal; PI: PISITTALA; qRTPCR: Quantitative real-time PCR; RIN: RNA integrity number; SA: Salicylic acid; SEM: Scanning electron microscope; Sp: Stamen petaloid; St: Stamen; TFs: Transcript factors; WGCNA: Weighted gene coexpression network analysis; WUS: WUSCHEL.

\section{References}

1. Wang Q, Zhang X. Colored illustration of lotus cultivars in China. Beijing: China Forestry Publishing House; 2005. 
2. Kubo N, Hirai M, Kaneko A et al. Development and characterization of simple sequence repeat (SSR) markers in the water lotus (Nelumbo nucifera). Aquat Bot. 2009, 90(2):191-194.

3. Meyerowitz EM, Smyth DR, Bowman JL. Abnormal flowers and pattern formation in floral development. Development. 1989, 106(2):209-217.

4. Irish VF. Evolution of petal identity. J Exp Bot. 2009, 60(9):2517-2527.

5. Bowman JL, Smyth DR, Meyerowitz EM. Genetic interactions among floral homeotic genes of Arabidopsis. Development. 1991, 112(1):1-20.

6. Coen ES, Meyerowitz EM. The war of the whorls: genetic interactions controlling flower development. Nature. 1991, 353(6339):31-37.

7. Dodsworth S. Petal, sepal, or tepal? B-genes and monocot flowers. Trends Plant Sci. 2017, 22(1):810.

8. Nakamura T, Fukuda T, Nakano $\mathrm{M}$ et al. The modified ABC model explains the development of the petaloid perianth of Agapanthus praecox ssp. orientalis (Agapanthaceae) flowers. Plant Mol Biol. 2005, 58(3):435-445.

9. Huang Z, Shi T, Zheng B et al. APETALA2 antagonizes the transcriptional activity of AGAMOUS in regulating floral stem cells in Arabidopsis thaliana. New phytol. 2017, 215(3):1197-1209.

10. Yanofsky MF, Ma H, Bowman JL et al. The protein encoded by the Arabidopsis homeotic gene agamous resembles transcription factors. Nature. 1990, 346(6279):35-39.

11. Dubois A, Raymond $\mathrm{O}, \mathrm{Maene} \mathrm{M}$ et al. Tinkering with the $\mathrm{C}$-function: a molecular frame for the selection of double flowers in cultivated roses. PLoS One. 2010, 5(2):e9288-e9288.

12. Tanaka Y, Oshima Y, Yamamura T et al. Multi-petal cyclamen flowers produced by AGAMOUS chimeric repressor expression. Sci Rep. 2013, 3:2641-2641.

13. Galimba KD, Tolkin TR, Sullivan AM et al. Loss of deeply conserved C-class floral homeotic gene function and $\mathrm{C}$ - and E-class protein interaction in a double-flowered ranunculid mutant. Proc Natl Acad Sci USA. 2012, 109(34):E2267-E2275.

14. Bao X, Franks B, Levin $\mathrm{J}$ et al. Repression of AGAMOUS by BELLRINGER in floral and inflorescence meristems. Plant Cell. 2004, 16:1478-1489.

15. Lenhard M, Bohnert A, Jurgens $\mathrm{G}$ et al. Termination of stem cell maintenance in Arabidopsis floral meristems by interactions between WUSCHEL and AGAMOUS. Cell. 2001, 105(6):805-814.

16. Wu M-F, Sang Y, Bezhani S et al. SWI2/SNF2 chromatin remodeling ATPases overcome polycomb repression and control floral organ identity with the LEAFY and SEPALLATA3 transcription factors. Proc Natl Acad Sci USA. 2012, 109(9):3576-3581.

17. Lin Z, Damaris RN, Shi T et al. Transcriptomic analysis identifies the key genes involved in stamen petaloid in lotus (Nelumbo nucifera). BMC Genomics. 2018, 19(1):554.

18. Lin Z, Liu M, Damaris RN et al. Genome-wide DNA methylation profiling in the lotus (Nelumbo nucifera) flower showing its contribution to the stamen petaloid. Plants-Basel. 2019, 8(5):135. 
19. Wang BQ, Liu J, Chu $L$ et al. Exogenous promoter triggers APETALA3 silencing through RNA-directed DNA methylation pathway in Arabidopsis. Int J Mol Sci. 2019, 20(18).

20. Ma N, Chen W, Fan TG et al. Low temperature-induced DNA hypermethylation attenuates expression of RhAG, an AGAMOUS homolog, and increases petal number in rose (Rosa hybrida). BMC Plant Biol. 2015, 15.

21. Zhang HM, Lang ZB, Zhu JK. Dynamics and function of DNA methylation in plants. Nat Rev Mol Cell Biol. 2018, 19(8):489-506.

22. Bräutigam K, Cronk Q. DNA methylation and the evolution of developmental complexity in plants. Front Plant Sci. 2018, 9:1447.

23. Lin Z, Zhang C, Cao D et al. The latest studies on lotus (Nelumbo nucifera)-an emerging horticultural model plant. Int J Mol Sci. 2019, 20(15).

24. Ming R, VanBuren R, Liu Y et al. Genome of the long-living sacred lotus (Nelumbo nucifera Gaertn.). Genome Biol. 2013, 14(5):R41-R41.

25. Wang $Y$, Fan G, Liu Y et al. The sacred lotus genome provides insights into the evolution of flowering plants. Plant J. 2013, 76(4):557-567.

26. Reeves $\mathrm{PH}$, Ellis $\mathrm{CM}$, Ploense SE et al. A regulatory network for coordinated flower maturation. PLoS Genet. 2012, 8(2):e1002506.

27. Reyes-Olalde Jl, Zúñiga-Mayo VM, Serwatowska J et al. The bHLH transcription factor SPATULA enables cytokinin signaling, and both activate auxin biosynthesis and transport genes at the medial domain of the gynoecium. PLoS Genet. 2017, 13(4):e1006726-e1006726.

28. Hudson KA, Hudson ME. The basic helix-loop-helix transcription factor family in the sacred lotus, Nelumbo Nucifera. Trop Plant Biol. 2014, 7(2):65-70.

29. Zhang $X$, Zhou $Y$, Ding $L$ et al. Transcription repressor HANABA TARANU controls flower development by integrating the actions of multiple hormones, floral organ specification genes, and GATA3 family genes in Arabidopsis. Plant Cell. 2013, 25(1):83-101.

30. Ding $L$, Yan $S$, Jiang $L$ et al. HANABA TARANU (HAN) Bridges Meristem and Organ Primordia Boundaries through PINHEAD, JAGGED, BLADE-ON-PETIOLE2 and CYTOKININ OXIDASE 3 during Flower Development in Arabidopsis. PLoS Genet. 2015, 11(9):e1005479.

31. Tanaka S, Mochizuki N, Nagatani A. Expression of the AtGH3a gene, an Arabidopsis homologue of the soybean GH3 gene, is regulated by phytochrome B. Plant Cell Physiol. 2002, 43(3):281-289.

32. Ohmiya A, Oda-Yamamizo C, Kishimoto S. Overexpression of CONSTANS-like 16 enhances chlorophyll accumulation in petunia corollas. Plant Sci. 2019, 280:90-96.

33. Liu Z, Boachon B, Lugan R et al. A conserved cytochrome P450 evolved in seed plants regulates flower maturation. Mol Plant. 2015, 8(12):1751-1765.

34. Adamski NM, Anastasiou E, Eriksson $S$ et al. Local maternal control of seed size by KLUH/CYP78A5dependent growth signaling. Proc Natl Acad Sci USA. 2009, 106(47):20115-20120. 
35. Anastasiou E, Kenz S, Gerstung M et al. Control of plant organ size by KLUH/CYP78A5-dependent intercellular signaling. Dev Cell. 2007, 13(6):843-856.

36. Rius SP, Casati P, Iglesias AA et al. Characterization of Arabidopsis lines deficient in GAPC-1, a cytosolic NAD-dependent glyceraldehyde-3-phosphate dehydrogenase. Plant Physiol. 2008, 148(3):1655-1667.

37. Dubos C, Stracke R, Grotewold E et al. MYB transcription factors in Arabidopsis. Trends Plant Sci. 2010, 15(10):573-581.

38. Heijmans K, Morel P, Vandenbussche M. MADS-box genes and floral development: the dark side. J Exp Bot. 2012, 63(15):5397-5404.

39. Tiancong $\mathrm{Q}$, Huang $\mathrm{H}$, Susheng $\mathrm{S}$ et al. Regulation of jasmonate-mediated stamen development and seed production by a bHLH-MYB complex in Arabidopsis. Plant Cell. 2015, 27(6):1620-1633.

40. Drews GN, Bowman JL, Meyerowitz EM. Negative regulation of the Arabidopsis homeotic gene AGAMOUS by the APETALA2 product. Cell. 1991, 65(6):991-1002.

41. Tzeng T-Y, Chen $\mathrm{H}-\mathrm{Y}$, Yang $\mathrm{C}-\mathrm{H}$. Ectopic expression of carpel-specific MADS box genes from lily and lisianthus causes similar homeotic conversion of sepal and petal in Arabidopsis. Plant Physiol. 2002, 130(4):1827-1836.

42. Heijmans K, Ament K, Rijpkema AS et al. Redefining C and D in the petunia ABC. Plant Cell. 2012, 24(6):2305-2317.

43. Liu Z, Zhang D, Liu D et al. Exon skipping of AGAMOUShomolog PrseAG in developing double flowers of Prunus lannesiana (Rosaceae). Plant Cell Rep. 2013, 32(2):227-237.

44. Zhu B, Li H, Wen $\mathrm{J}$ et al. Functional specialization of duplicated AGAMOUS homologs in regulating floral organ development of Medicago truncatula. Front Plant Sci. 2018, 9:854-854.

45. Lampugnani ER, Kilinc A, Smyth DR. PETAL LOSS is a boundary gene that inhibits growth between developing sepals in Arabidopsis thaliana. Plant J. 2012, 71(5):724-735.

46. Sablowski R. Control of patterning, growth, and differentiation by floral organ identity genes. J Exp Bot. 2015, 66(4):1065-1073.

47. Rebocho AB, Kennaway JR, Bangham JA et al. Formation and shaping of the Antirminum flower through modulation of the CUP boundary gene. Curr Biol. 2017, 27(17):2610-2622.e2613.

48. Lu S, Song T, Kosma D et al. Arabidopsis CER8 encodes Long-Chain Acyl CoA Synthetase 1 (LACS1) and has overlapping functions with LACS2 in plant wax and cutin synthesis. Plant J. 2009, 59:553564.

49. Kim D, Pertea G, Trapnell C et al. TopHat2: accurate alignment of transcriptomes in the presence of insertions, deletions and gene fusions. Genome Biol. 2013, 14(4):R36.

50. Trapnell C, Williams BA, Pertea $G$ et al. Transcript assembly and quantification by RNA-Seq reveals unannotated transcripts and isoform switching during cell differentiation. Nat Biotechnol. 2010, 28(5):511-515. 
51. Li B, Dewey CN. RSEM: accurate transcript quantification from RNA-Seq data with or without a reference genome. BMC Bioinformatics. 2011, 12(1):323.

\section{Figures}
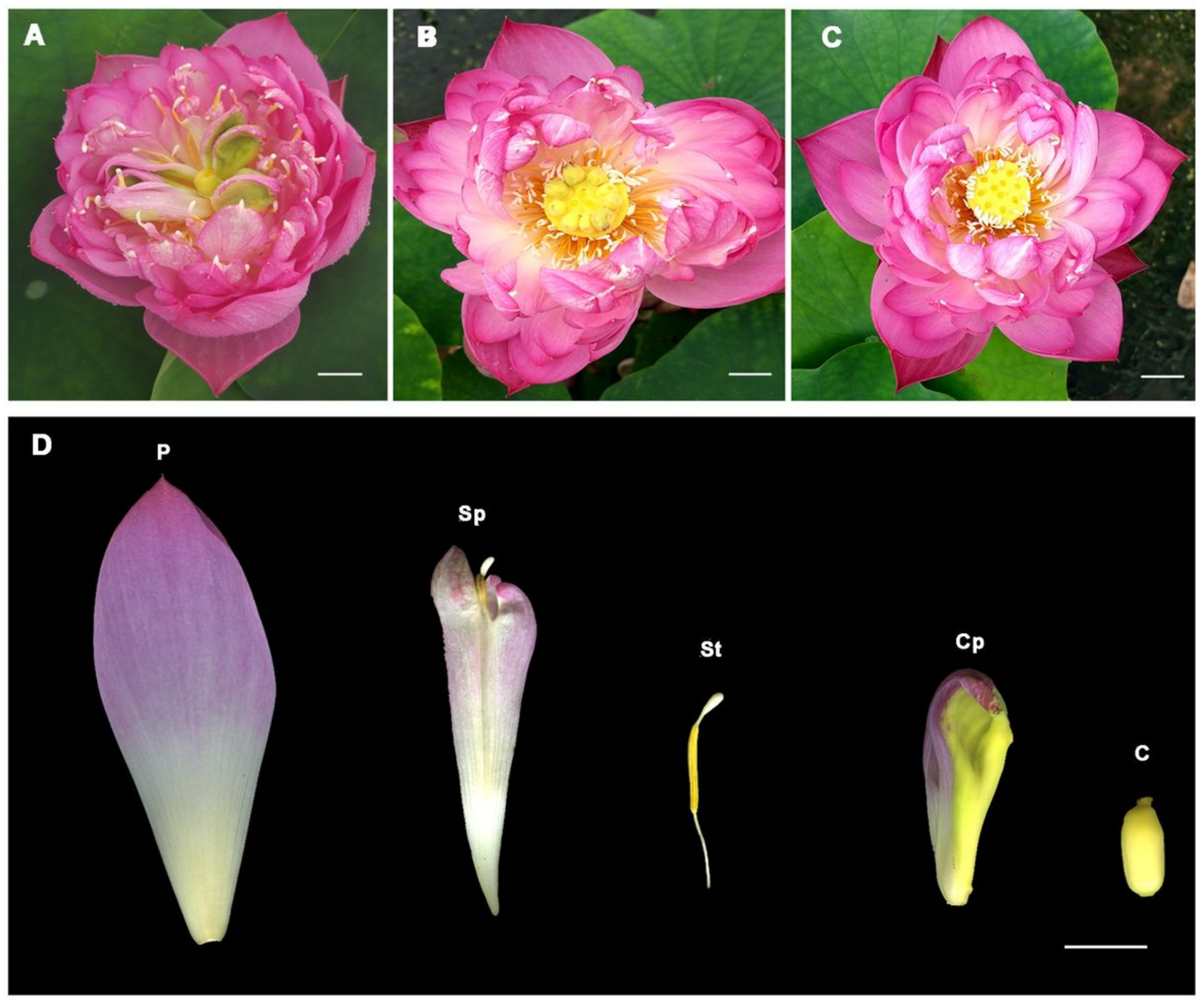

\section{Figure 1}

The flower of sacred lotus 'Sleeping Beauty' (A-C) The different flower morphology of 'Sleeping Beauty' was imaged. (D) Five different floral organs, including petal ( $P$ ), stamen petaloid (Sp), stamen (St), carpel petaloid (Cp), and carpel (C). Bars are all $1 \mathrm{~cm}$. $P$ was collected from flowers showed in A and $C$, and then mixed together for next study; St and $\mathrm{C}$ were collected following the same mothed of P collection. Sp and Cp were collected form flowers showed in A. 


\section{SEM}

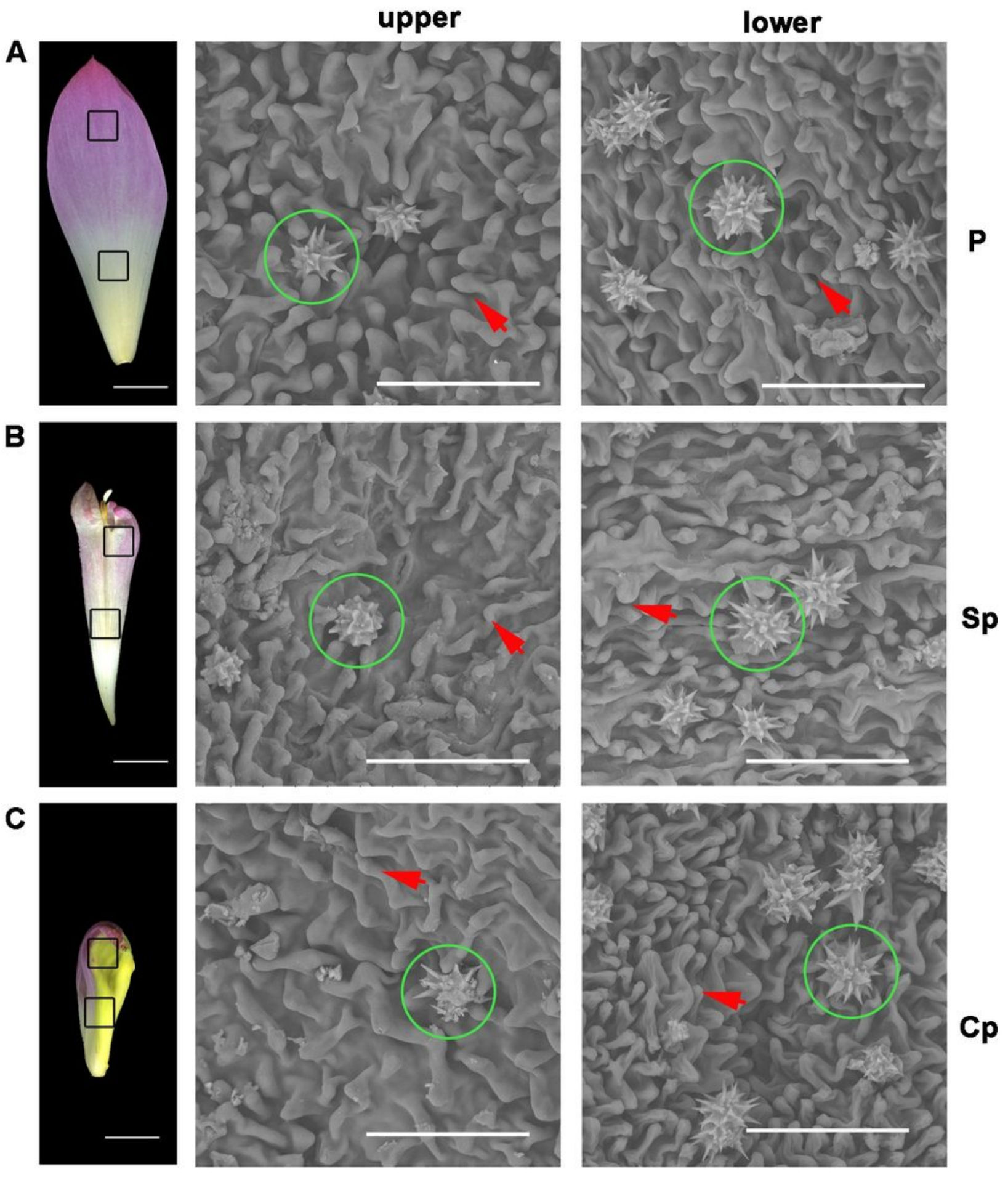

\section{Figure 2}

Scanning electron microscope (SEM) observation of epidermal structure of the petal-like organ in lotus (A) Epidermal cells of upper and lower of petal (P). (B) Epidermal cells are in upper and lower of stamen petaloid (Sp). (C) Epidermal cells of upper and lower carpel petaloid (Cp). Bars of SEM are all $50 \mu \mathrm{m}$. Mastoid cell are shown by arrow and wax crystals are marked in green circle. 
A

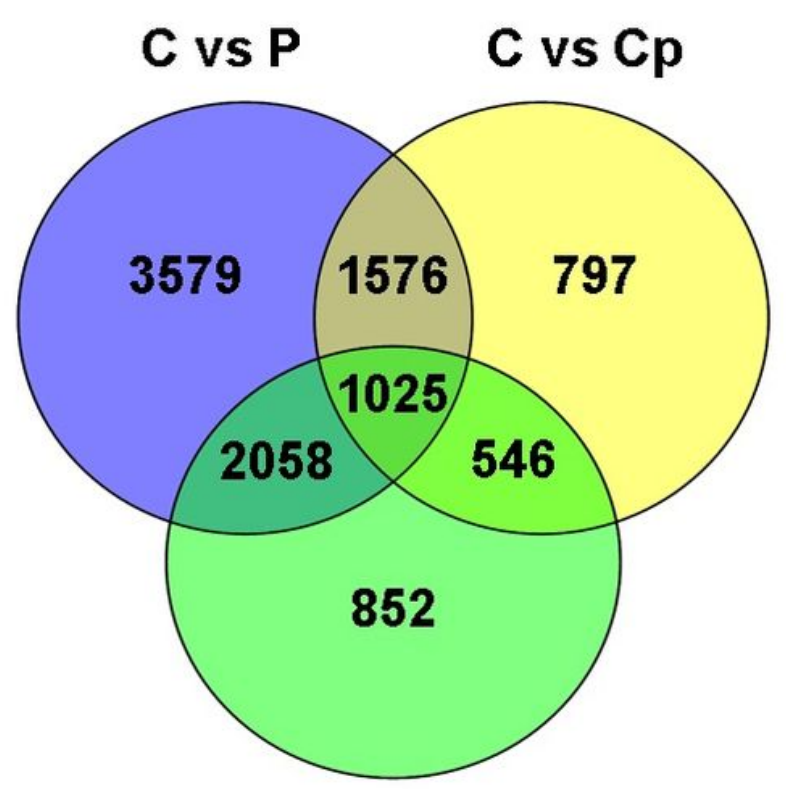

P vs Cp

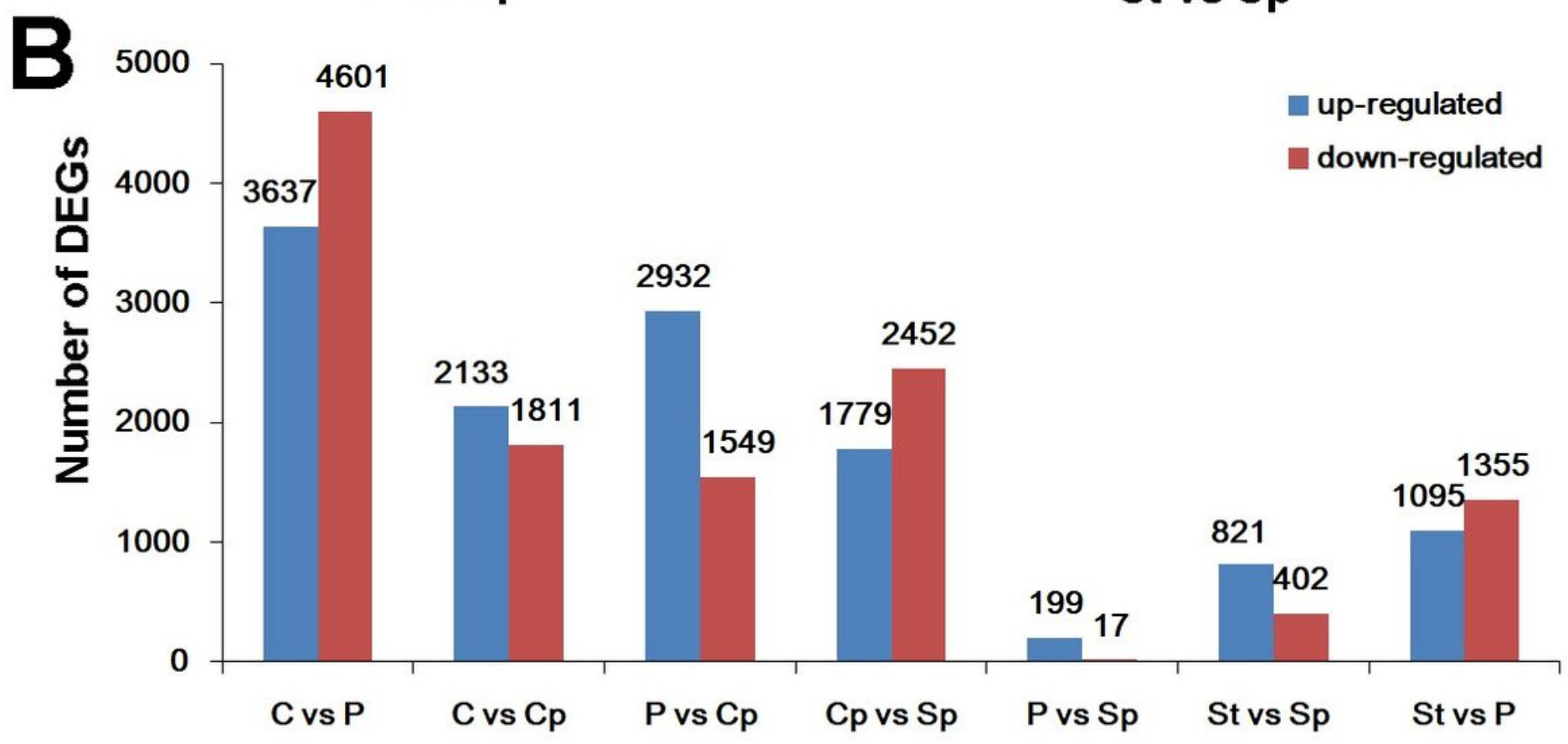

Figure 3

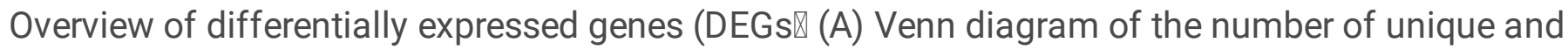
common DEGs in the two comparisons ( $P$ vs Sp, St vs P, and St vs Sp; C vs P, C vs Cp, and P vs Cp). (B) The number of up-regulated and down-regulated DEGs in the two comparisons ( $P$ vs Sp, St vs $P$, and St vs Sp; C vs P, C vs Cp, and P vs Cp). The y-axis represents the number of genes and the $x$-axis represents the different comparison. 

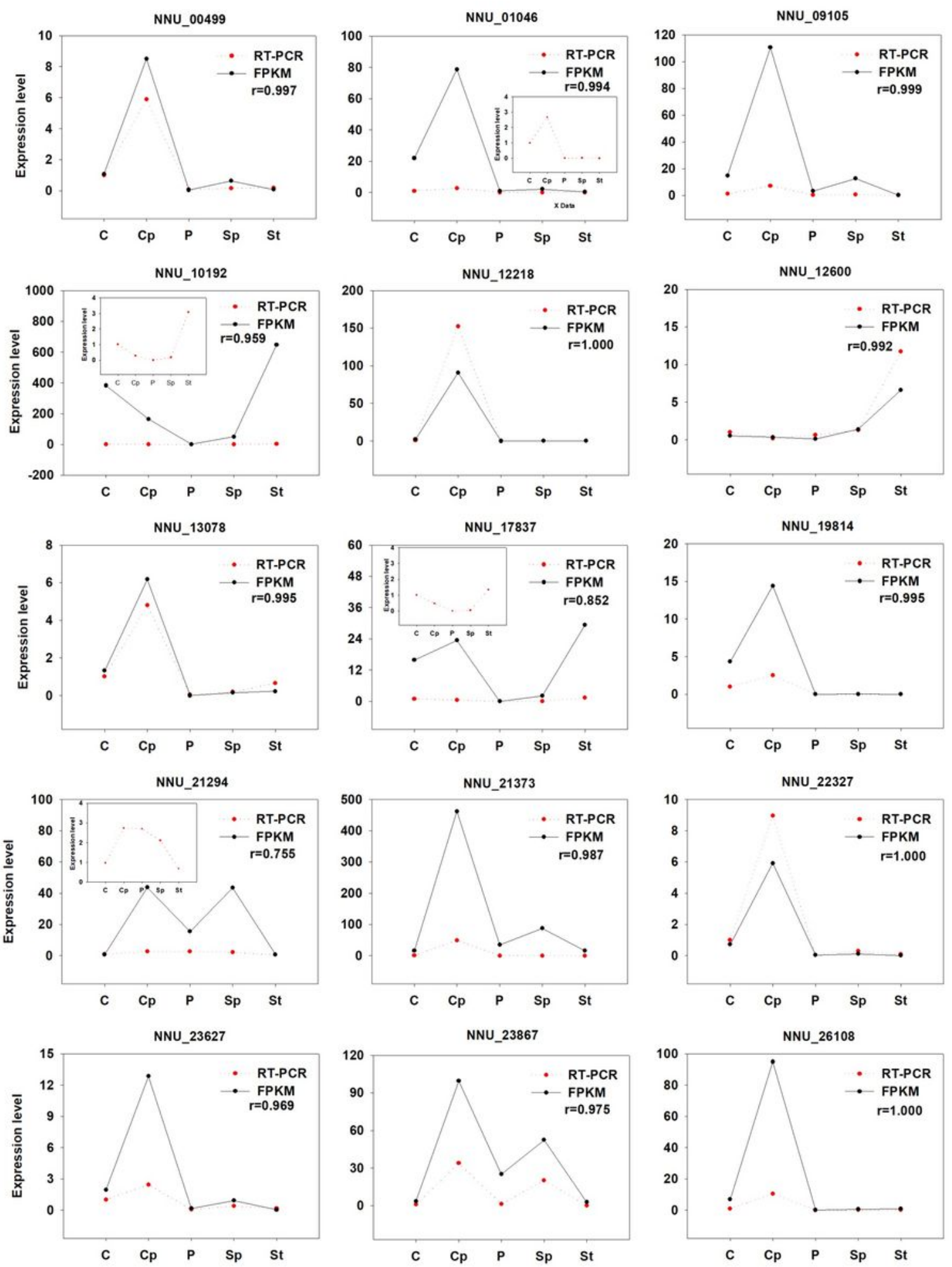

Figure 4

Validation of RNA-seq data using qRT-PCR. The relationship ( $r$ ) with FPKM values and relative expression levels of qRT-PCR assays is shown. RT-PCR was show by red and FPKM is represented by black. 
A
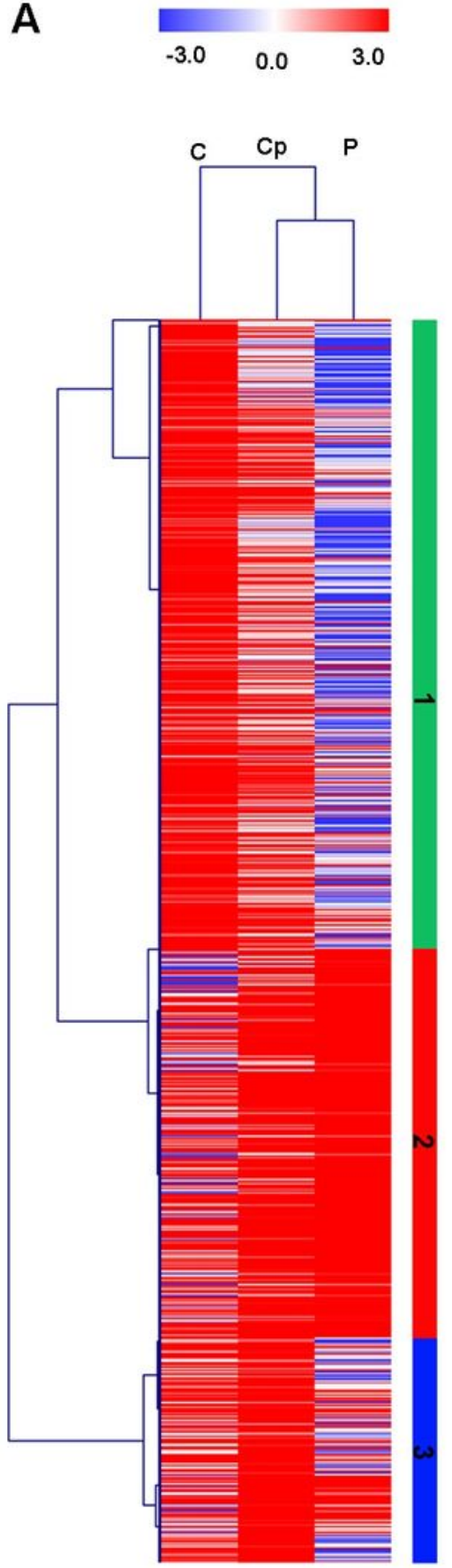

B

Plus-end-directed microtubule motor activity Microtubule binding Ciliary cytoplasm Microtubule Plant-type cell wall Motile cilium methylation-dependent chromatin silencing

\section{톯} Cell proliferation Intraciliary anterograde transport Oxidation-reduction process Chromatin silencing by small RNA

Phragmoplast assembly Cilium organization Seed development

Phragmoplast microtubule organization Regulation of G2/M transition of mitotic cell cycle DNA replication initiation Regulation of DNA replication Histone H3-K9 methylation DNA methylation 0.0

C

plant-pathogen interaction Arginine and proline metabolism Cysteine and methionine metabolism Glycine, serine and threonine metabolism Histidine metabolism
Lysine degradation Phenylalanine metabolism Phenylalanine, tyrosine and tryptophan biosynthesis ryptophan metabolism Valine, leucine and isoleucine degradation Anthocyanin biosynthesis
Flavonoid biosynthesis Phenylpropanoid biosynthesis Ascorbate and aldarate metabolism Galactose metabolism Galactose metabolism
Glycolysis / Gluconeogenesis Glyoxylate and dicarboxylate metabolism
Inositol phosphate metabolism Glyoxylate and dicarboxylate metabolism
Inositol phosphate metabolism Pyruvate metabolism Starch and sucrose metabolism
in photosynthetic organisms Photosynthesis
Photosynthesis-antenna proteins Sulfur metabolism Biosynthesis of amino acids Carbon metabolism Fatty acid degradation Fatty acid elongation Glycerolipid metabolism phospholipid metabolism Sphingolipid metabolism alpha-Linolenic acid metabolism hyrin and chlorophyll metabolism Cyanoamino acid metabolism Glutathione metabolism
beta-Alanine metabolism Brassinosteroid biosythesis Carotenoid biosynthesis Limonene and pinene degradation Terpenoid backbone biosynthesis Phosphatidylinositol signaling system Plant hormone signal transduction Endocytosis Peroxisome Protein processing in endoplasmic reticulum Ubiquitin mediated proteolysis Ribosome

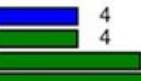

The Most enriched GO Terms

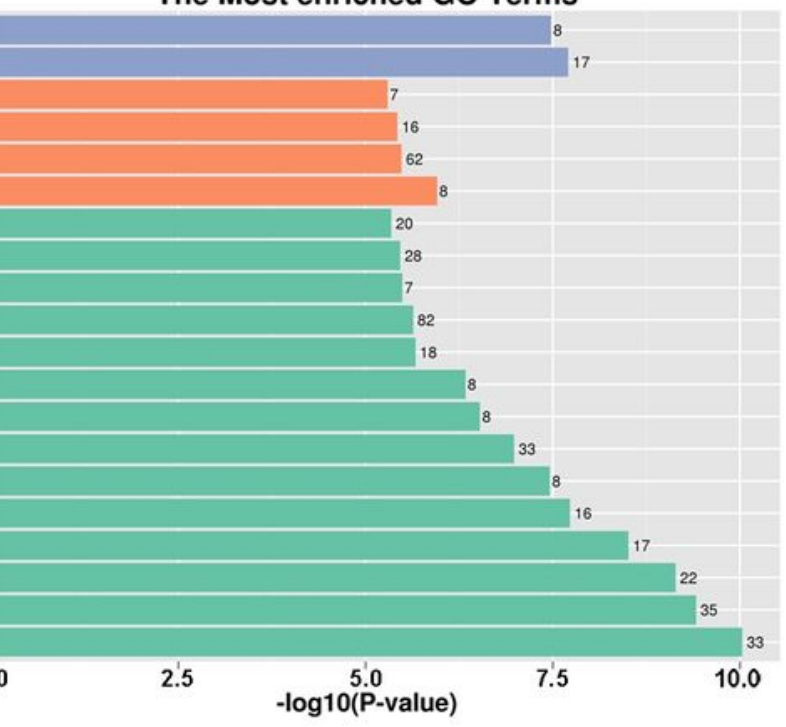

o10(P-value)

Organismal Systems

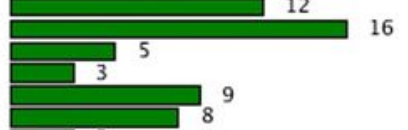

\section{6}
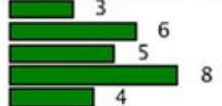

3

$\square$

$\square^{4}$

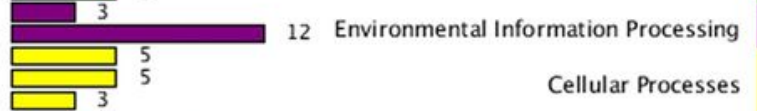

$0 \%$

Annotated Genes

\section{Figure 5}

Hierarchical clustering analysis (A), enriched Go terms (B), and KEGG classification (C) of 812 annotated DEGs among $\mathrm{P}, \mathrm{Cp}$, and $\mathrm{C}$. 

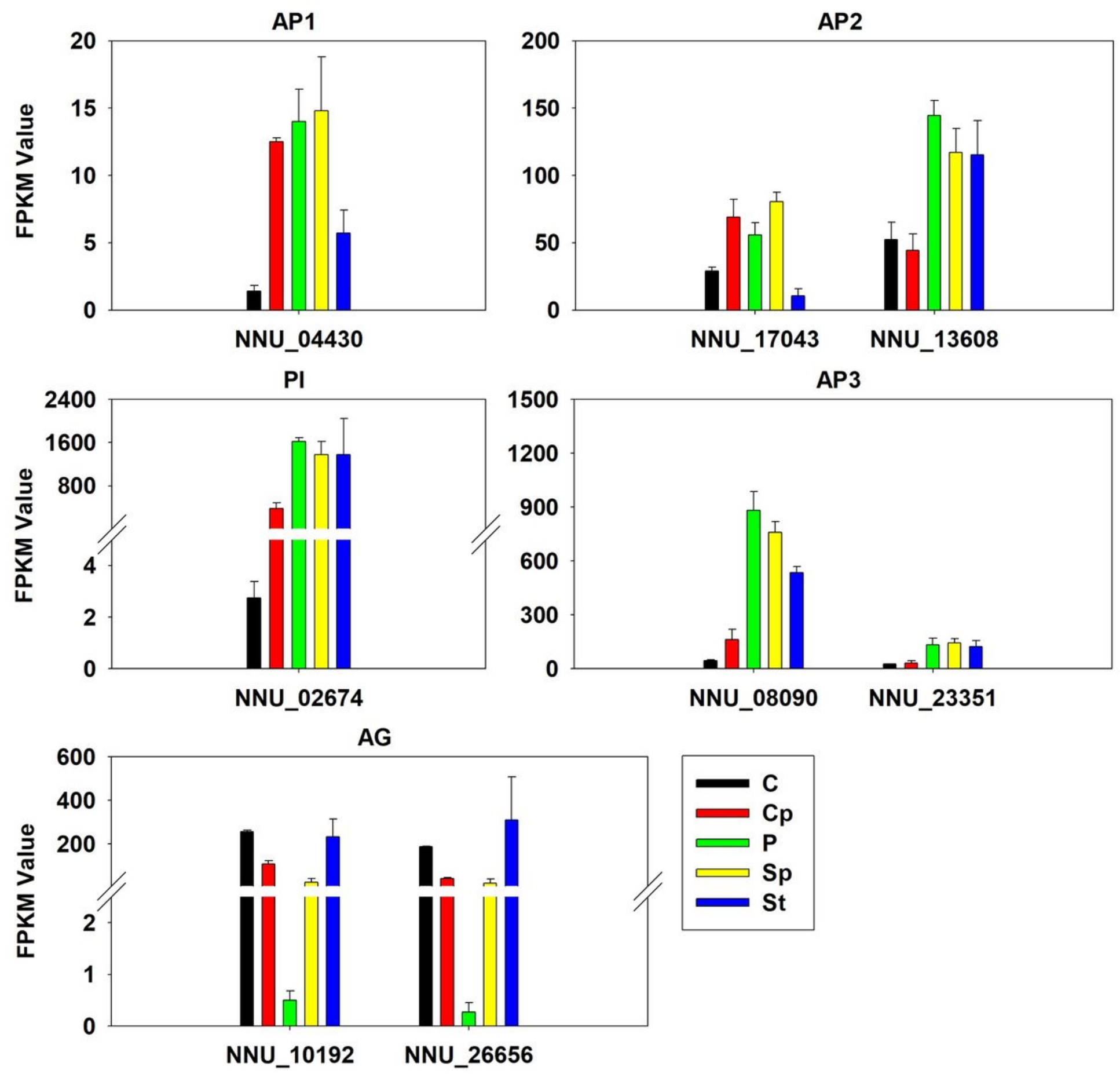

Figure 6

The expression pattern of $A B C$ model genes in lotus. Values represent the means and standard errors (SEs) of three biological replicates. Each sample was performed by real-time PCR validates with triplicate. The expression level of each gene in one of three carpels was arbitrarily set as 1.0 
Module-trait relationships

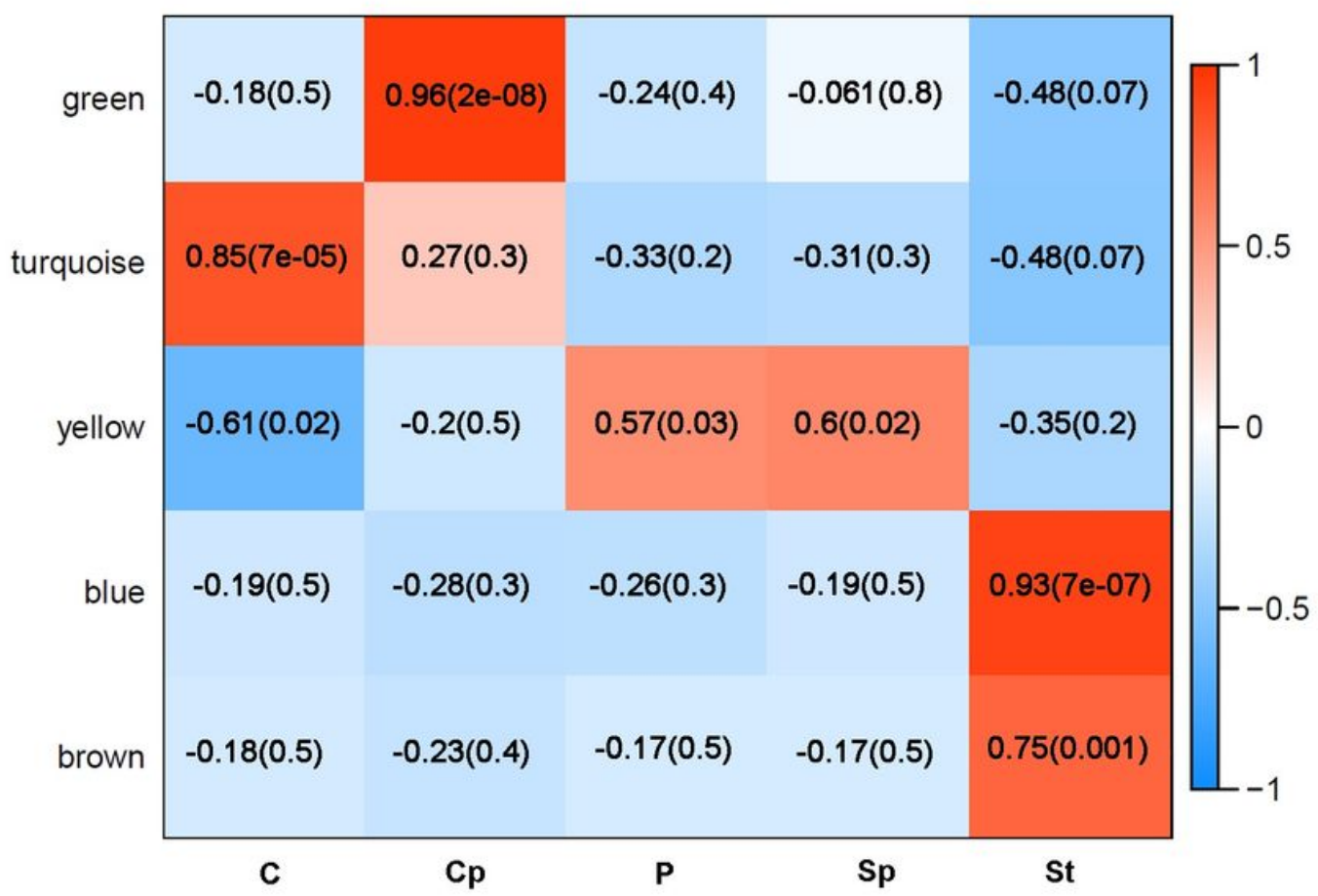

B
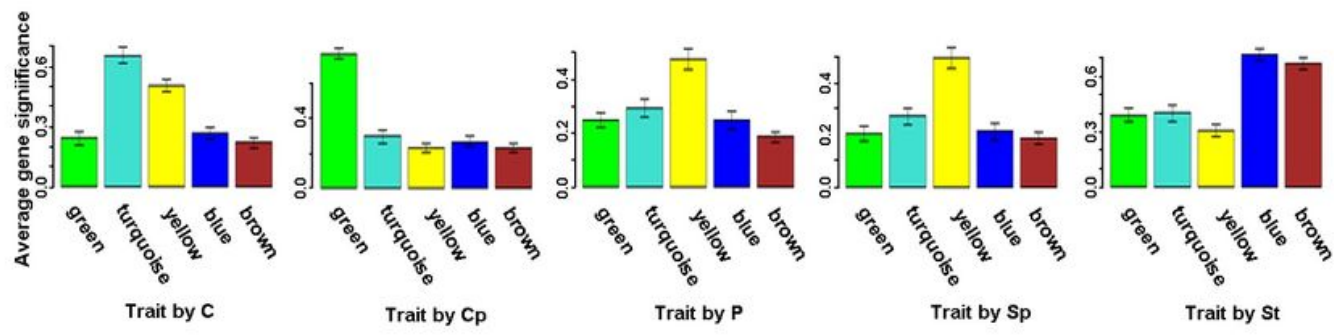

C

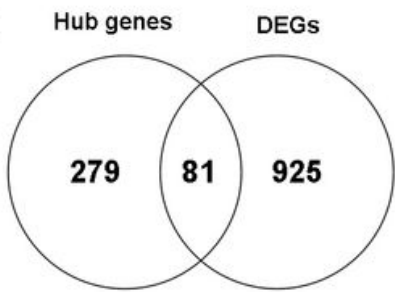

D $\quad \begin{array}{lll}-5.0 & 0.0 & 5.0\end{array}$

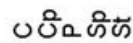

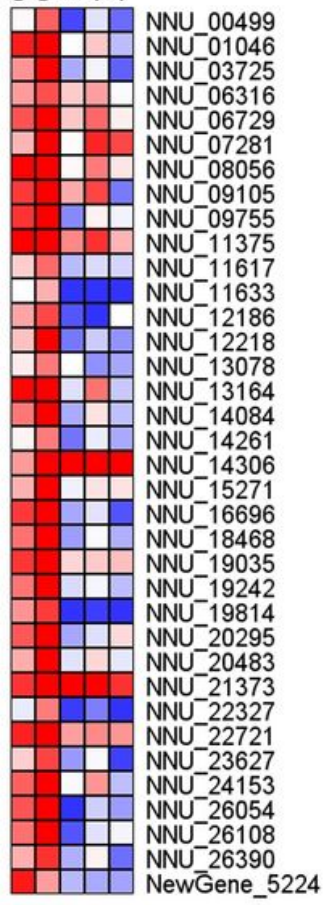

Figure 7

WGNCA of the lotus tissues (A) Module trait relationships. (B) Gene trait significance. (C) Venn of hub genes and DEGs. (D) Heat map of 37 hub DEGs (log2FC $>5$ or $<-5)$. The color scale of the heat map ranges from saturated blue (value, -5.0 ) to saturated red (value, 5.0 ) in the natural logarithmic scale. 

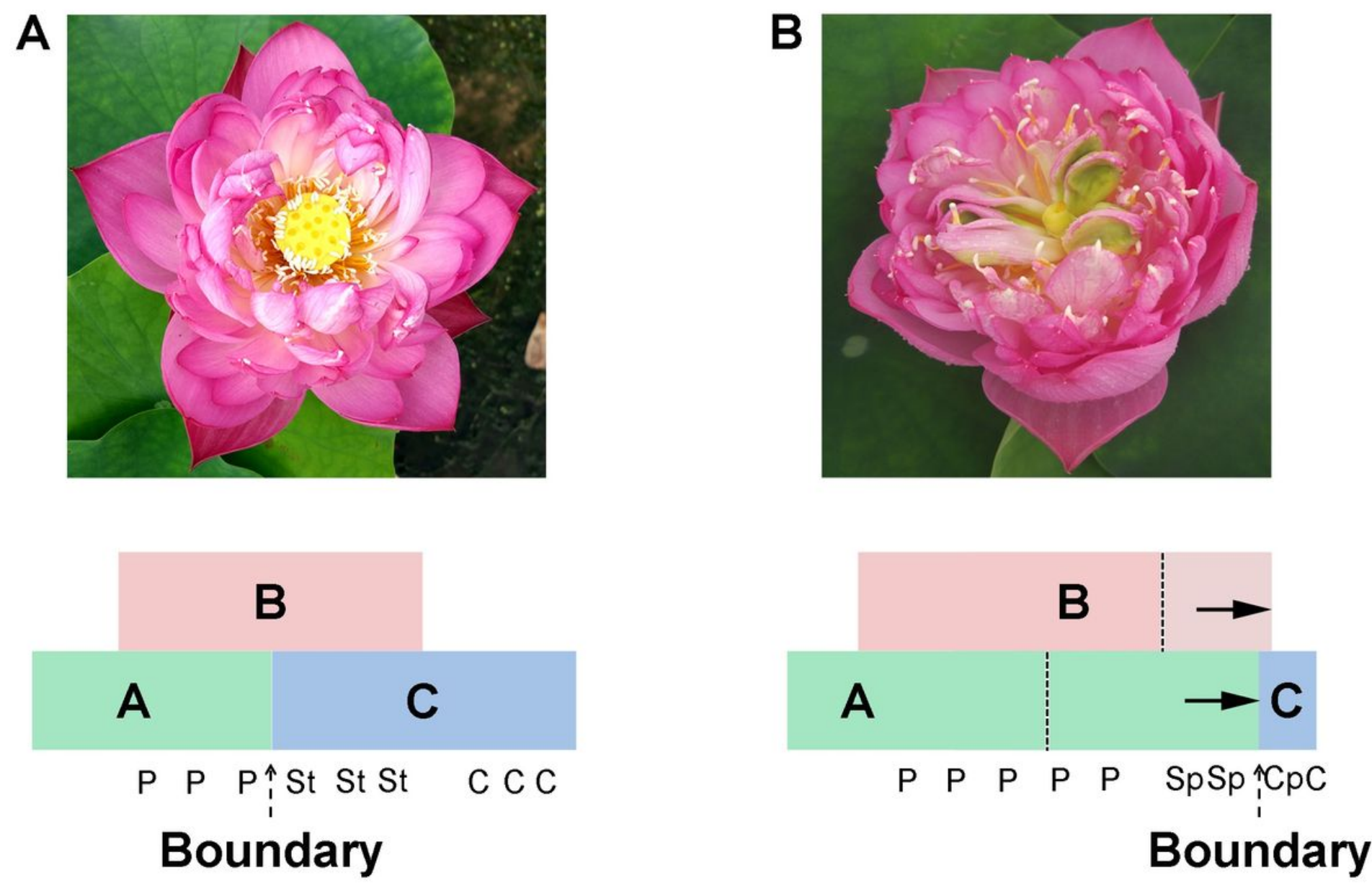

Figure 8

The proposed model in flower pattern of lotus. (A) Normal flower pattern in lotus. (B) Aberrant flower pattern in lotus. The petals/stamens/carpels boundaries are slide in the flowers.

\section{Supplementary Files}

This is a list of supplementary files associated with this preprint. Click to download.

- Fig.S1.tif

- Fig.S2.tif

- Fig.S3.tif

- Fig.S4.tif

- Fig.S5.tif

- TableS1.docx

- TableS2.xlsx

- Tables3.xIsx

- Tables4.docx 